This is a postprint version of the following published document:

E. Prior, C. de Dios, R. Criado, M. Ortsiefer, P. Meissner and P. Acedo. (2016). Dynamics of dual-polarization VCSEL-based optical frequency combs under optical injection locking. Photonics Technology Letters, 41 (17), pp. 4083-4086

DOI: https://doi.org/10.1364/OL.41.004083

(C) 2016 Optical Society of America 


\title{
Dynamics of dual-polarization VCSEL-based optical frequency combs under optical injection locking
}

\author{
E. Prior, ${ }^{1}$ C. de Dios, ${ }^{1, *}$ R. Criado, ${ }^{2}$ M. Ortsiefer, ${ }^{3}$ P. Melssner, ${ }^{4}$ and P. Acedo ${ }^{1}$ \\ 'Eectronics Technology Department, Universidad Carlos III de Madrid, Leganés 28911, Spain \\ ${ }^{2}$ Luz Wavelabs, S.L. Av. Gregorio Peces Barba, Leganés 28919, Spain \\ 'Vertilas GmbH, Garching, Germany \\ 'Technische Universität Darmstadt, Damnstadt, Germany \\ "Corresponding author: odios Qinguçmes
}

The present experimental work studies the dynamics of dual-polarization optical frequency combs (OFCs) based on gain switching (GS) vertical-cavity surface-emitting laser (VCSEL) diodes under optical injection locking (OIL). This study presents two main results. First, we have obtained an overall comb formed by two orthogonally polarized sub-combs with comparable span and power. The overall comb shows enhanced optical span and flatness and high coherence between its modes. The second result is that we have been able to control the polarization state of the overall comb by tuning the polarization state of the injected light by locking the same single teeth of the comb. This produces an over all comb with single polarization that is parallel or orthogonal These are novel findings that provide for the development of efficient and compact OFCs based on GS VCSEL sources with versatile polarization dynamios. 2016 Optical Society of America

OCIS codes: (250.7260) Vertical cavity surface emitting lasers; (230.0250) Optoelectronics; (260.5430) Polarization; (1403520) Lasers, injection-locked.

In recent years, optical frequency combs (OFCs) have attracted enormous attention in many scientific disciplines [ [1 5 ]. Among the available optical comb technologies, OFCs based on laser diodes (LDs) offer suitable combs with competitive costs and efficiency [G]. Therefore, the use of vertical cavity surface emitting laser (VCSEL) diodes for OFC generation takes this advantage even further, in terms of compactness, low cost, and low energy consumption and opens the possibility of mass production [7]. Very recently, we reported high quality OFCs with record optical span and enengy efficiency using VCSEL diode technology and gain switching (GS) modulation, a floxible technique to generate highly coherent frequency tunable combs [8]. However, further efforts are required to improveOFCs based on VCSELs (VCSELOFC), especially to extend the optical span they offer while maintaining the ad vantages they have al ready demonstrated; such an optical source would be useful in the fields of $\mathrm{THz}$ generation [4] or green optical communications [9].

Optical injection locking (OIL) in LDs has been commonly used to improve the performance of the emitted light, and is typically focused on laser spectral narrowing, frequency chirp reduction, noise reduction, and modulation bandwidth en hancement [10]. These have also been demonstrated with VCSEL sources [11]. This technique consists of the injection of light from an external source, the master laser, into the device called the slave laser. Under certain conditions, the output light of the slave laser locks with the injected light and acquires the same frequency and phase characteristics. OIL techniques have also been used to modify and enhance OFCs [12].

In view of the interest in VCSEL OFCs and the versatility of the OIL techniques, their combination may result in a new comb with enriched characteristics. We have studied this com bination and evaluated the behavior of VCSEL OFCs under the OIL regime with the objective of improving the OFCs based on this $L D$ technology.

However, the interest in VCSEL OFCs goes further, as they have a unique feature that makes their behavior under OIL noteworthy. In previous works [13], we observed that the combs shaped using the LD technology are formed by two sub combs with orthogonal polarizations. One of these combs is related to the main mode of the VCSEL and the other, to the suppressed mode, present in these types of devices. We ob served that these two orthogonally polarized combs are strongly phase correlated due to the GS modulation, and we suggested that an enhanced dual polarization OFC formed by two equiv alent coherent combs with orthogonal polarization could be obtained by balancing their relative power using OIL. The present work shows that this is possible using special OIL tech niques where the polarization of the master bser diode is con trolled to perform parallel or orthogonal injection [14]. Recent studies have evaluated the singular behavior of VCSELs under polarization controlled injection [15]. 
This study presents two main results combining VCSEL OFCs GS techniques and polarization controlled OIL. The first result is that we obtained an overall comb with enhanced optical span and flatness, and high coherence between the modes formed by two orthogonally polarized sub combs with comparable span and power. This dual polarization OFC will find application in several fields, for example, ultrafast laser dy namics [16] or polarization division multiplexing optical com munication [17]. The second result is that we found a way to controlling the polarization state of the overall comb by tuning the polarization of the injected light to produce an enhanced single polarization comb that is parallel or orthogonal. All of the results have been obtained by controlling the polarization state of the master laser and performing injection always in the same teeth of the initial OFC with the same power ratio. These are novel findings that allow for the development of efficient and compact OFC sources with high coherence and versatile polari zation dynamics.

The VCSEL device used in this work is a state of the tech nology device (VERTILAS model VL 1550 8G P2 H4) with a $P_{x}$ mode lasing at $1537.95 \mathrm{~nm}$ with $4.2 \mathrm{dBm}$ peak power and an orthogonal $P_{y}$ mode at $1538.20 \mathrm{~nm}$ with $47.3 \mathrm{dBm}$. The optimum comb, in terms of span and flatness, was obtained with the device stabilized at $25^{\circ} \mathrm{C}$ with a bias current of $27 \mathrm{~mA}$ and an input RF signal to produce the GS regime of $19 \mathrm{dBm}$ at $5 \mathrm{GHz}$. The ratios describing the GS operation are $I_{\mathrm{RF}} / I_{\text {bizs }}=$ 2.1 and $I_{\text {biz }} / I_{\text {th }}=1.7$. This VCSEL OFC is shown in Fig. 2(a). The Ith of our LD has increased to $16 \mathrm{~mA}$ after extensive experimental work. Therefore, the GS operation to achieve an optical comb equivalent to the one shown in pre vious works has also varied [8]. The master laser is a discrete mode (DM) laser [18]. The DM laser was set to $19^{\circ} \mathrm{C}$, to fall in the VCSEL OFC optical span, and the biasing current was set to $38 \mathrm{~mA}$. To change the emission wavelength of the master to match the slave and achieve the injection, this current will be slightly tuned.

In Fig. 1 we present our experimental setup. The master path starts with the DM laser emitting in a continuous wave $(\mathrm{CW})$. Then, the signal is attenuated with a variable optical attenuator (VOA) and a 50/50 optical coupler (OC1) to adjust

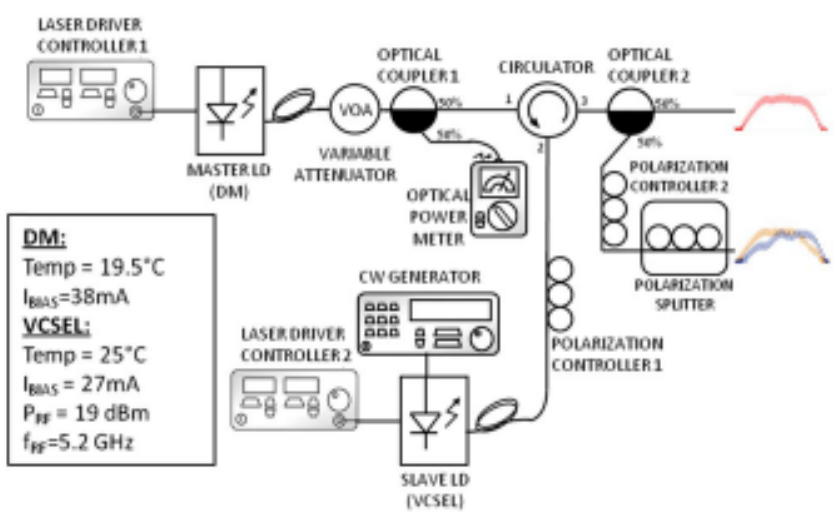

Fig. 1. Experimental setup. The comb is generated inside the VCSEL $\left(25^{\circ} \mathrm{C}, 27 \mathrm{~mA}, 5.2 \mathrm{GHz}, 19 \mathrm{dBm}\right)$ at the same time it is optically injected by the DM laser $\left(19.5^{\circ} \mathrm{C}, 38 \mathrm{~mA}\right)$ through a circu lator. The injection ratio is $6.6 \mathrm{~dB}$. The optical output is first power divided and then one arm is split into two orthogonally polarized sub combs with a PS. See text for more details. the appropriate injected power to the slave, which will be monitored with a power meter placed in one of the OC out puts. The other OC output will enter the circulator to reach the slave optical parh.

The slave path is formed by the VCSEL and a polarization controller (PC1), which selects the polarization of the ingoing master light to the light being generated inside the cavity of the VCSEL. This polarization adjustment is critical to achieve OIL. However, in our case, it is even more important because it makes it possible to select the polarization of the light entering the slave to perform parallel or orthogonal polarization injec tion locking. The master light coming from the circulator enters the VCSEL and then the output signal goes through the circulator to the output path, which is connected to the measurement equipment. We measured the optical spectra in an optical spectrum analyzer (OSA) with $0.002 \mathrm{~nm}$ resolution and the electrical spectra after the linewidth setup in an elec trical spectrum analyzer (ESA), using an ultrafast $50 \mathrm{GHz}$ photodetector. The temporal trace will be measured in an autocorrelator which has an erbium doped fiber amplifier (EDFA) and a PC in its entrance.

In order to separate and study the polarization components of the output comb, we included a second 50/50 coupler (OC2) to divide the optical output. One branch will be used to evaluate the overall comb under injection locking, OIL OFC. The other branch of OC2 has a second polarization controller (PC2) and a polarization splitter (PS) to discriminate between the parallel and orthogonal sub combs. With this setup, we obtained the traces in Fig. 2(c).

In Fig. 2 we show the aforementioned OFCs and some other significant optical traces. Figure 2(a) presents the VCSEL OFC which is the VCSEL output when there is no OIL. This comb has 25 teeth in the $20 \mathrm{~dB}$ span, which cor responds to $130 \mathrm{GHz}$. We know from previous work that this VCSEL OFC is formed by two sub combs, one main comb with parallel polarization [13] and the residual one with orthogonal polarization and much lower power. This sub comb is observed in the small hip in the upper wavelengths in the VCSEL OFC, and one goal of this work is to power up this residual with OIL. The DM master laser CW light is also traced in Fig. 2.

In the first injection experiment, we evaluated how OIL can influence the characteristics of the overall comb and its com ponents. In order to do so, we optimized the injection fre quency and polarization and the injection ratio. When the master DM source is tuned for emission at $1541.63 \mathrm{~nm}$ and the injection ratio is $6.6 \mathrm{~dB}$, the OIL is optimum in terms of the span and flatness of the overall comb [Fig. 2(b)]. This OIL OFC is broader, with 27 teeth in the $20 \mathrm{~dB}$ span corre sponding to $140 \mathrm{GHz}$. It is also more symmetric and flatter than the VCSEL OFC. In this case, the polarization of the in jected light from the master was carefully tuned using the PC1 in order to equalize the optical power levels of the parallel and orthogonally polarized sub combs. At the output of the PS, we observed two orthogonally polarized sub combs. One of them included the lower wavelengths, which we call $\mathrm{OIL}_{\mathrm{X}} \mathrm{OFC}$ [Fig. 2(c), trace with the same name] and the second one, called $\mathrm{OIL}_{Y}$ OFC [Fig. 2(c), OIL $\mathrm{I}_{Y}$ OFC trace] corresponds to the upper part of the total comb, which is orthogonally po larized. Therefore, the OIL is capable of balancing the power of both sub combs and increasing the orthogonal modes. 


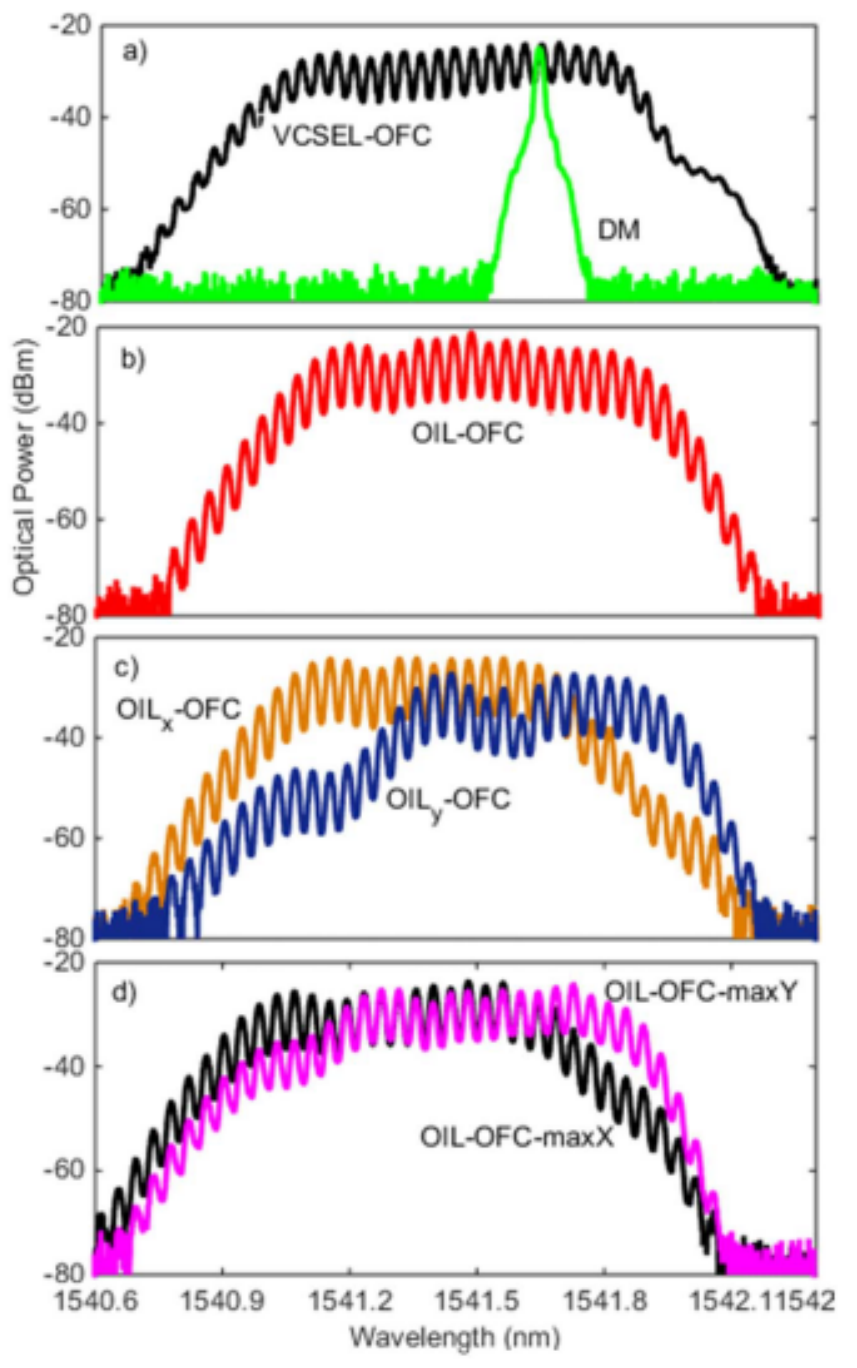

Fig. 2 (a) VCSEL OFC trace shows the output of the VCSEL with GS. This is a comb with 25 teeth in the $20 \mathrm{~dB}$ span which corresponds to $130 \mathrm{GHz}$. DM trace is the master light being injected into the VCSEL at $1541.63 \mathrm{~nm}$. (b) OIL OFC output signal with optical in jection locking adjusting the polarization to equalize both sub combs. The resulting comb has 27 teeth in the $20 \mathrm{~dB}$ span, which corresponds

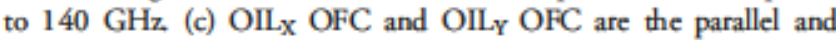
orthogonal polarization components of the OIL OFC, respectively. This means that the injection is used to balance the sub combs with different polarizations to exhibit similar optical powers and span. (d) OIL OFCs adjusting the master polarization to maximize either the parallel (OIL OFC maxX) or the orthogonal comb (OIL OFC maxY). See text for details.

Therefore, an enhanced dual polarization VCSEL based OFC can be generated.

In the second optical injection experiment, we evaluated the influence of the state of polarization of the injected light on the total OIL OFCs [14]. For this purpose, we used PC1 to change the polarization state of the master laser and then observed the output comb and the polarization components. In Fig. 2(d), we show the injected optical comb where the parallel or main comb has been maximized, OIL OFC maxX. This was achieved when the polarization of the injected light coincided with that of this parallel sub comb. In this case, the orthogonal

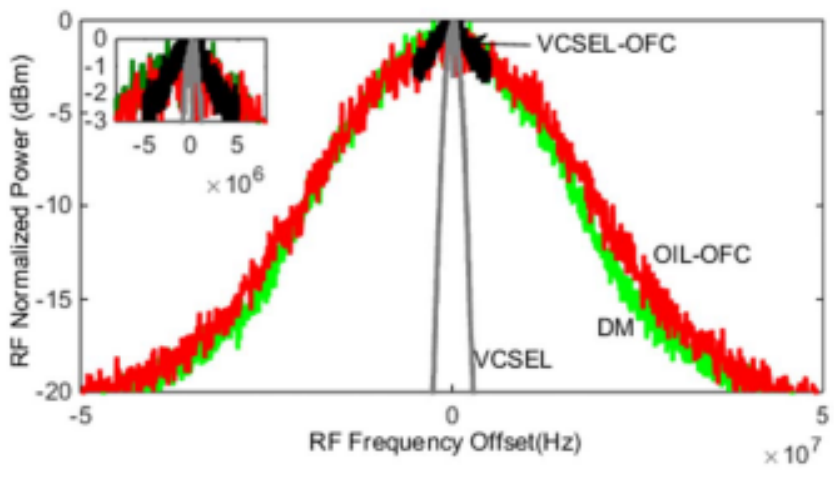

Fig. 3. Electrical spectra after self heterodyning. The linewidths are half the $3 \mathrm{~dB}$ bandwidth shown in the traces. VCSEL in CW (VCSEL trace), which has an optical linewidth of $1 \mathrm{MHz}, \mathrm{DM}$ in CW (DM trace) with an optical linewidth of $7 \mathrm{MHz}$, VCSEL OFC with an op tical linewidth of $4 \mathrm{MHz}$, and OIL OFC with an optical linewidth of $8 \mathrm{MHz}$. See text for details.

comb was suppressed and the polarization of the final comb switched to be entirely parallel. The OIL OFC maxY trace in Fig. 2(d) shows the output comb when the polarization of the master laser coincides with that of the orthogonal sub comb. Then, the initially residual comb turns to be the main and only comb, also achieving total polarization switching. These two traces are limited examples, and the OIL OFC in Fig. 2(b) will be halfway, obtaining a balance between both polarization states.

It is important to remark on the difference between the re sults of these two injection experiments. In Fig. 2(c) we show the two components that form the OIL OFC in Fig. 2(b). On the other hand, in Fig. 2(d), we adjust the polarization to enhance only one linear state of polarization while suppressing the other one. This implies that by controlling the polarization of the master light injected in the VCSELs, we can balance the sub combs with different polarizations to exhibit similar optical powers and span, or we can maintain one of them while can celling the other one, switching the polarization state of the resulting optical comb.

In addition to the optical spectra, we measured the optical linewidth of the different signals previously shown. For this purpose, we used the delayed self heterodyne interferometric technique [19] and obtained the electrical spectra shown in Fig. 3, where the optical linewidths are half of the $3 \mathrm{~dB}$ band width of the plotted lines. This image shows the linewidth of VCSEL when working with $C W$ emission, which is $1 \mathrm{MHz}$, and the VCSEL OFC linewidth increases to $4 \mathrm{MHz}$ due to the GS regime. The DM linewidth is $7 \mathrm{MHz}$. The injected mode in the OIL OFC is slightly broader and has a linewidth of $8 \mathrm{MHz}$. As expected, the master characteristics are inherited by the slave and the linewidth of the modes in the final comb is another parameter that can be controlled using the OIL technique.

Finally, we also obtained the autocorrelation traces of the temporal pulses generated with and without the OIL scheme. The autocorrelation trace (ACT) of the VCSEL OFC and the ACT of the OIL OFC are shown in Fig. 4. The full width at half maximum (FWHM) of the ACT, without injection, is equal to $18.3 \mathrm{ps}$. When the optimum point of $\mathrm{OIL}$ is achieved [Fig. 2(c)], this width is reduced to $11.8 \mathrm{ps}$. These ACT traces 


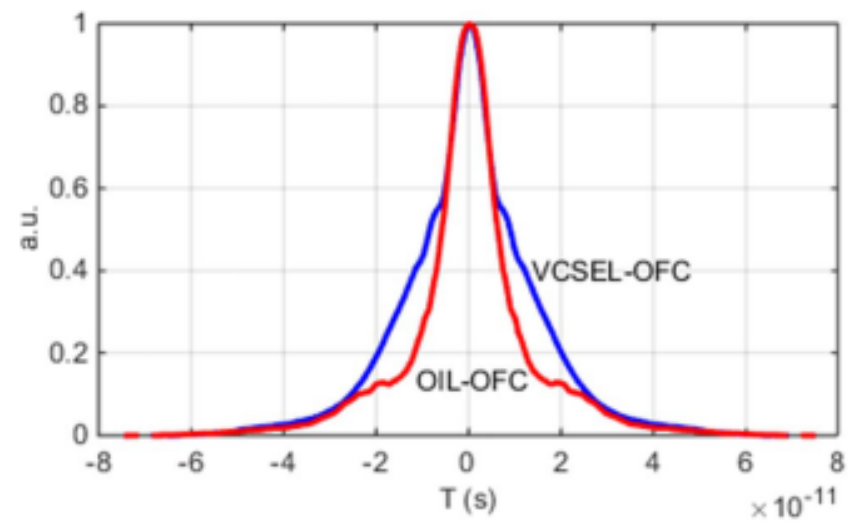

Fig. 4. Autocorrelation traces. VCSEL OFC with 18.3 ps FWHM and OIL OFC with $11.8 \mathrm{ps}$ FWHM. The OIL technique reduces the width of the ACT traces and changes the shape, which reduces podestals.

are complex and exhibit different shapes with pedestals. This is typical of the GS technique, and such traces must be analyzed using time retrieval algorithms [19] and metrics such as the root mean square time bandwidth product: $\mathrm{TBP}_{\text {rms }}=\omega_{\text {rms }} \cdot \tau_{\text {rms }}$, where $\omega_{\text {rms }}$ is the rms width of the optical spectra and $\tau_{\text {rms }}$ is the rms pulse width. Regardless of the pulse shape or spectral structure, the fundamental limit of $\mathrm{TBP}_{\text {rms }}$ is 0.5 [20]. In this case, the TBP ${ }_{\text {rms }}$ for the VCSEL OFC is equal to 2.34 and for the OIL OFC is goes down to 2.02. Though the result is still far from the fundamental limit, the injection technique also has a positive impact in the temporal profile of the source.

In condusion, we have shown our latest results in VCSEL OFCs in which we have evaluated the polarization dynamics of these combs under optical injection to improve the charac teristic of these types of sources. We have observed that the control of the polarization of the injected light dearly influences the overall optical comb and the sub combs of which it is formed. We have been able to balance and equalize the power associated with those sub combs, obtaining an enhanced dual polarization OFC. In addition, we have also tuned the in jected polarization to cancel one of these sub combs, inducing a polarization switch along all the modes of the overall comb. This allows for control of the polarization state of the final comb, which is quite a remarkable result that extends the ver satility and benefits of VCSEL devices for OFC generation.
Funding. Ministerio de Economía y Competitividad (MINECO) (RTC 20142661 7, TEC 201452147 R).

\section{REFERENCES}

1. T. Udem, R. Holzwarth, and T. W. Hânsch, Nature 416, 233 (2002).

2. C. Chen, C. Zhang, D. Lu, K. Qiu, and S. Lu, Opt. Lett. 37, 3954 (2012).

3. P. J. Delfyett, I. Ozdur, N. Hoghooghi, M. Akbulut, J. Davila Rodriguez, and S. Bhooplapur, IEEE J. Sel. Top. Quantum Electron. 18, 258 (2012).

4. A. R. Criado, C. de Dios, E. Prior, G. H. Dohler, S. Preu, S. Matzer, H. Lu, A. C. Gossard, and P. Acedo, IEEE Trans. Terahertz Sci. Technol. 3, 461 (2013).

5. P. Martin Mateos, M. Ruiz Llata, J. Posada Roman, and P. Acedo, IEEE Photon. Technol. Lett. 27, 1309 (2015).

6. P. M. Anandarajah, R. Maher, Y. Q. Xu, S. Latkowski, J. O'Carroll, S. G. Murdoch, R. Phelan, J. O'Gorman, and L P. Barry, IEEE Photon. J. 3, 112 (2011).

7. E. Kapon and A. Sirbu, Nat. Photonics 3, 27 (2009).

8. E. Prior, C. de Dios, A. R. Criado, M. Ortsiefer, P. Meissner, and P. Acedo, IEEE Photon. Technol. Lett. 26, 1 (2014).

9. R. S. Tucker, IEEE J. Sel. Top. Quantum Electron. 17, 245 (2011).

10. E. K. Lau and M. C. Wu, IEEE J. Sel. Top. Quantum Electron. 15, 618 (2009).

11. L Chrostowski and C. J. Chang Hasnain, IEEE J. Sel. Top. Quantum Electron. 9, 1386 (2003).

12. E. Sooudi, C. de Dios, J. G. Mcinemey, H. Huyet, L. Lelarge, K. Merghem, R. Rosales, A. Martinez, A. Ramdane, and S. P. Hegarty, IEEE J. Sel. Top. Quantum Electron. 19, 1101208 (2013).

13. E. Prior, C. De Dios, M. Ortsiefer, P. Meissner, and P. Acedo, J. Lightwave Technol. 33, 4572 (2015).

14. A. Quirce, P. Perez, H. Lin, A. Valle, L Pesquera, K. Panajotov, and H. Thienpont, IEEE J. Quantum Electron. 50, 921 (2014).

15. A. Quirce, P. Pérez, A. Popp, Á. Valle, L Pesquera, Y. Hong, H. Thienpont, and K. Panajotov, Opt. Lett. 41, 2664 (2016).

16. M. Brunel, J. Thévenin, M. Vallet, J. Thevenin, and M. Vallet, in CLEO (2013), paper CF1G.5.

17. A. D. Shiner, M. Reimer, A. Borowiec, S. O. Gharan, J. Gaudette, P. Mehta, D. Chartton, K. Roberts, and M. O'Sullivan, Opt. Express 22, 20366 (2014).

18. B. Kelly, R. Phelan, D. Jones, C. Herbert, J. O'Carroll, M. Rensing, J. Wendelboe, C. B. Watts, A. Kaszubowska Anandarajah, P. Perry, C. Guignard, L. P. Barry, and J. O'Goman, Electron. Lett. 43, 1282 (2007).

19. T. Okoshi, K. Kikuchi, and A. Nakayama, Electron. Lett. 16, 630 (1980).

20. R. Trebino, Frequency Resolved Optical Gating: The Measurement of Ulitrashort Laser Pulses (Springer, 2000). 